\title{
Fondement et organisation des réseaux européens de conservation des ressources génétiques forestières
}

\author{
M Arbez \\ Institut national de la recherche agronomique, station de recherches forestières \\ de Bordeaux-Cestas, BP 45, 33611 Gazinet Cedex, France
}

Résumé - La première conférence ministérielle sur la protection des forêts en Europe, réunie à Strasbourg en 1990, a engagé 32 pays signataires à initier ou à poursuivre une politique concertée de conservation des ressources génétiques forestières et mis en place une structure provisoire de suivi. Cette résolution s'est concrétisée par une enquête internationale sur la situation actuelle des ressources génétiques forestières en Europe et un inventaire des mesures techniques et réglementaires prises aujourd'hui dans ce but. Elle s'est accompagnée de la mise en place des 4 premiers réseaux-pilotes de conservation concernant l'épicéa (Picea abies), le chêne liège (Quercus suber), le peuplier noir (Populus nigra) et les feuillus précieux (Rosacées principalement). Chaque espèce ou groupe d'espèces représente un modèle différent de répartition spatiale et de système de reproduction. La seconde conférence ministérielle sur la protection des forêts, tenue à Helsinki en juin 1993, a validé ces orientations et proposé la création d'un programme coopératif européen permanent sous l'égide de l'IBPGR et du département forestier de la FAO. Des recherches restent nécessaires sur la structure de la variabilité génétique, les mécanismes d'entretien de cette variabilité en forêts naturelles et les méthodes d'aménagement et de sylviculture permettant d'obtenir l'adaptabilité maximum.

ressource génétique / forêt / réseau / coopération internationale / Europe

Summary - Basis and organization of the European forest tree gene conservation networks. The first ministerial conference on the protection of forests in Europe, held in Strasbourg in 1990, engaged 32 signatory countries to initiate or to follow a concerted policy for forest tree gene conservation and to establish a provisional monitoring structure. This resolution has resulted in an international survey on the present status of forest tree genetic resources in Europe and relevant technical and regulatory measures. In parallel the first 4 pilot gene conservation networks concerning Norway spruce (Picea abies), Cork oak (Quercus suber), black poplar (Populus nigra) and the noble hardwoods (Rosaceaes essentially) were established. Each species or group of species represents a distinct model of spatial distribution and mating system. The second ministerial conference on the protection of forests, held in Helsinki in June 1993, has confirmed these orientations and has proposed the creation of a permanent European cooperative programme under the aegis of the International Board for Plant Genetic Resources and the forest department of the Food 
and Agriculture Organization. Further research is necessary on the structure of the genetic variability, the mechanisms to maintain this variability in natural forests, and management and sylvicultural methods able to generate maximum adaptability.

genetic resource / forest / network / international cooperation / Europe

\section{INTRODUCTION}

En Europe, la prise de conscience croissante des problèmes d'environnement et la place privilégiée qu'y tiennent souvent les forêts, sont à l'origine d'une demande sociale forte en faveur de leur préservation et de la conservation des ressources génétiques forestières (Arbez, 1984).

Les stratégies mises en œuvre s'expliquent par les particularités biologiques et génétiques des arbres forestiers et des connaissances scientifiques récentes encore très fragmentaires. Ce sont des espèces très longévives, leur durée de vie atteint ou même dépasse très largement le siècle. L'aire de répartition d'une espèce est généralement très vaste et atteint souvent l'échelle du continent européen. Les arbres forestiers sont allogames, en majorité diploïdes et monoïques, leur diversité génétique mesurée à partir des marqueurs moléculaires apparaît comme l'une des plus importantes rencontrées chez les organismes vivants (Hamrick et Godt, 1990). Contrairement aux plantes cultivées, les arbres forestiers demeurent encore à l'aube de leur domestication; si l'évolution de leur variabilité n'a pas échappé aux influences humaines, elle n'en a pas pour autant été encore bouleversée. Précédant la conférence de Rio au plan mondial, mais de manière concrète et plus contrôlable, la conférence de Strasbourg sur la protection des forêts en Europe a mis en œuvre une réflexion coordonnée et des réseaux de conservation des ressources génétiques forestières. Cet article fait une analyse rapide du contexte, des objectifs et des problématiques qui sous-tendent les stratégies mises en œuvre.

\section{L'HÉRITAGE DE LA CONFÉRENCE DE STRASBOURG (1990)}

La première conférence ministérielle sur la protection des forêts en Europe s'est tenue à Strasbourg en décembre 1990, à l'instigation initiale des ministres français et finlandais de l'agriculture, vite rejoints dans le comité d'organisation par le Portugal et la Pologne. Trente-deux pays ont participé à cette conférence et la majorité d'entre eux ont adopté 6 résolutions concourant à une meilleure protection des forêts. Seule la résolution 2 , consacrée à la conservation des ressources génétiques forestières, retiendra provisoirement notre attention.

Cette résolution, signée par tous les États participants, impliquait de leur part et en fonction des moyens disponibles, l'élaboration et la mise en œuvre d'une politique de conservation des ressources génétiques forestières. Rédigée en termes généraux, cette résolution justifie l'urgence des mesures à prendre, énumère quelques principes d'action et invite les États à la coopération internationale. 


\section{Des mesures d'urgence}

Même si les forêts tempérées apparaissent provisoirement moins menacées dans leur diversité génétique que les forêts tropicales, il y a cependant de nombreuses raisons d'intervenir rapidement :

- la régénération naturelle des forêts mélangées est progressivement remplacée par des plantations monospécifiques;

- les transferts géographiques des matériels forestiers de reproduction (semences, plants, boutures) sont devenus très fréquents;

- quelques espèces très productives, représentées chacune par un effectif toujours limité de variétés améliorées, sont communément utilisées en reboisement;

- en conséquence, les races ou les écotypes locaux a priori bien adaptés, sont menacés d'extinction ou pour le moins d'hybridations intempestives par les espèces ou les populations introduites;

- d'autres activités humaines font peser de lourdes menaces sur la diversité originelle des espèces forestières : l'urbanisation croissante et les aménagements de toutes sortes, barrages, routes et autoroutes, aires de stationnement, pistes de skis, aires de loisirs, les changements atmosphériques rapides provoqués par les émissions d'origine humaine (pluies acides et réchauffement climatique global).

\section{Des méthodes simples, économiques et efficaces à long terme}

Ces mesures sont les suivantes :

- l'urgence des mesures à prendre ne permet pas d'attendre toutes les certitudes scientifiques que l'on serait tenté d'exiger avant d'agir. Il faut arrêter un plan d'urgence avec les informations de tous ordres déjà disponibles aujourd'hui dans le domaine de la génétique, de l'écologie et de l'économie de chaque espèce forestière concernée;

- ces constatations, sont en faveur de méthodes simples, faciles à mettre en œuvre et efficaces sur le long terme; elles privilégient chaque fois qu'il est possible des mesures de conservation in situ des espèces forestières majeures, bien intégrées dans la gestion des forêts, et complétées lorsque c'est utile ou nécessaire par la conservation ex situ.

\section{Une stratégie de conservation intégrée, combinant mesures in situ et ex situ}

Dans la plupart des cas, la conservation in situ apparait comme une méthode économique, apte à garantir sur le long terme la conservation d'un échantillon représentatif de la diversité génétique d'une espèce donnée, sans le soustraire pour autant à l'évolution des pressions de sélection (maladies, insectes, adversités climatiques). Les simulations réalisées en France et en Finlande montrent que pour la plupart des espèces forestières sociales (celles vivant et se reproduisant en peuplements), la mise en réserve génétique in situ, d'un échantillon de surface inférieure à $1 \%$ des forêts de l'espèce considérée permet d'assurer convenablement la conservation. Les principales contraintes concernent la taille (10 ha), l'importance de la zone d'isolement (90 ha) et la régénération des placeaux de conservation à partir de semences rigoureusement autochtones (régénération naturelle ou complétée à partir de semences 
autochtones). Dans le strict respect de ce cahier des charges, par ailleurs mentionné dans le document d'aménagement de la forêt, rien ne semble actuellement s'opposer au maintien des éclaircies et de la production du bois.

Économique et efficace dans de très nombreux cas, la conservation in situ apparait cependant inadaptée ou insuffisante dans certaines situations :

- lorsque des modifications drastiques ou exagérément rapides de l'environnement ne permettent plus à l'espèce de survivre ou simplement de se reproduire, c'est déjà le cas dans certaines forêts d'Europe centrale, en raison des dommages causés par les pluies acides (Ziehe et al, 1987);

- si le pronostic de réchauffement climatique rapide se réalise, de nombreuses espèces incapables de migrer ou d'évoluer assez rapidement seraient condamnées à mourir sur place; les forêts boréales et d'altitude seraient les premières menacées;

- enfin, il existe de nombreuses espèces forestières disséminées par bouquets ou par individus isolés, dont la conservation in situ exigerait des surfaces trop considérables pour être réalisable.

Dans toutes ces situations difficiles, la conservation $e x$ situ apparaît préférable. En règle générale, elle possède aussi un grand intérêt pour l'amélioration génétique et la conservation dynamique. Dans la majorité des cas, c'est la combinaison des deux méthodes, modulée en fonction de la situation particulière de l'espèce, qui est recommandée.

\section{Les espèces secondaires ou rares}

Les espèces forestières majeures couvrent des surfaces importantes, elles ont un intérêt économique et écologique indiscutable et les États ne sont pas trop difficiles à convaincre pour financer les programmes de conservation. Il en va différemment des espèces secondaires ou mineures, pour lesquelles la seule perspective réaliste semble aujourd'hui résider dans la conservation d'un échantillon représentatif des écosystèmes forestiers qui les hébergent. Les espèces rares sont justiciables des mêmes mesures, ou d'une conservation ex situ si leur intérêt économique le permet.

\section{Biodiversité et gestion forestière}

Au-delà des mesures spécialisées, engagées à travers les réseaux de conservation, sur une fraction limitée du territoire, il faut encourager les méthodes d'aménagement et de sylviculture préservant ou accroissant la diversité génétique inter- et intraspécifique des forêts.

Dans l'immense majorité des cas, les services forestiers des États européens sont convaincus par ces recommandations et les mettent déjà en œuvre en forêt publique.

\section{Des structures nationales identifiées et des financements garantis}

Toute action de conservation n'a de sens qu'inscrite dans la continuité et dans la durée. Cette activité doit être le fait d'organismes nationaux identifiés et responsables, dotés de programmes pluri-annuels et de financements garantis sur le long terme. 


\section{Un instrument de coopération internationale à l'échelle européenne}

Compte tenu de l'ampleur de la répartition géographique de la plupart des espèces forestières et du caractère général de certaines menaces affectant leur diversité génétique (pluies acides, réchauffement climatique global, ou simplement évolution des méthodes d'aménagement et de sylviculture), il est clair que les programmes de conservation à long terme des Ėtats doivent être concertés, sinon coordonnés, pour être pleinement efficaces (Palmberg et Esquinas-Alcazar, 1990). Cette constatation milite en faveur d'une instance internationale de réflexion, d'animation et de coordination des réseaux européens de conservation des ressources génétiques forestières.

\section{Une procédure de suivi des résolutions}

Comparée à d'autres conférences, parfois plus prestigieuses comme celle de Rio, la conférence de Strasbourg s'est dotée de comités de suivi des résolutions prises (Collectif, 1993). D'essence à l'origine quadripartite (Finlande, France, Pologne, Portugal) ces comités ont été chargés de traduire les résolutions souvent générales sous forme de projets définis, scientifiquement argumentés et pratiquement réalisables. Le comité de suivi de la résolution 2 , concernant la conservation des ressources génétiques forestières, a fonctionné durant 2 ans et demi pour élaborer, commencer à mettre en œuvre et faire valider par les États un programme en 3 parties :

- une enquête sur l'état des ressources génétiques forestières européennes, les mesures techniques et réglementaires mises en œuvre pour leur conservation, et les priorités d'action;

- la conception et la mise en place des premiers réseaux pilotes de conservation;

- une réflexion et des propositions concernant le rôle et la nature d'une structure internationale d'animation et de coordination des réseaux de conservation des ressources génétiques forestières en Europe.

\section{LA SITUATION ACTUELLE DES RESSOURCES GÉNÉTIQUES FORESTIÈRES EUROPÉENNES}

Pour valider ou modifier les premiers choix d'espèces pour les réseaux de conservation et orienter les suivants en fonction des priorités, une analyse de la situation actuelle des ressources génétiques forestières s'imposait.

Cet examen d'ensemble a été réalisé en lançant une enquête auprès des responsables nationaux de la mise en œuvre de la résolution 2 . Un questionnaire concernant à la fois l'inventaire des espèces menacées dans leur existence ou leur diversité génétique, les mesures d'étude génétique et de conservation à long terme dont elles sont déjà l'objet, et plus généralement la politique de conservation et les dispositions réglementaires qui en résultent, a été élaboré à Rome en mars 1992 par la commission de suivi, avec la collaboration de la FAO (Food and Agriculture Organisation of the United Nations), de l'IBPGR (International Board for Plant Genetic Resources) et d'un représentant de la Commission des Communautés européennes.

Vingt-quatre pays sur les 29 interrogés ont répondu à l'enquête. L'analyse des réponses a fourni les résultats résumés au tableau I (fig 1 et Collectif, 1993). 
Tableau I. Enquête internationale sur l'état des ressources génétiques forestières : programmes d'études et de conservation en Europe (Collectif, 1993).

\begin{tabular}{lc}
\hline Espèces forestières & Pourcentage \\
\hline Abies alba & 4,2 \\
Abies spp & 3,7 \\
Acer platanoides & 1,0 \\
Acer pseudoplatanus & 2,6 \\
Alnus glutinosa & 2,1 \\
Alnus incana & 1,0 \\
Betula pendula & 1,6 \\
Betula pubescens & 1,6 \\
Castanea sativa & 1,0 \\
Cupressus sempervirens & 1,0 \\
Fagus sylvatica & 5,2 \\
Fraxinus excelsior & 2,1 \\
Juglans regia & 1,0 \\
Larix decidua & 3,7 \\
Picea abies & 9,9 \\
Picea cembra & 2,1 \\
Picea sitchensis & 1,0 \\
Pinus halepensis & 1,6 \\
Pinus mugo & 1,6 \\
Pinus nigra & 4,7 \\
Pinus pinaster & 1,6 \\
Pinus pinea & 1,0 \\
Pinus sylvestris & 7,9 \\
Populus alba & 1,0 \\
Populus nigra & 1,6 \\
Populus tremula & 1,6 \\
Prunus avium & 2,1 \\
Pseudotsuga menziesii & 3,1 \\
Quercus robur/pedunculata & 6,8 \\
Quercus rubra & 1,0 \\
Quercus ssp & 3,7 \\
Robinia pseudoacacia & 1,0 \\
Salix ssp & 2,6 \\
Sorbus ssp & 2,6 \\
Taxus bacata & 1,0 \\
Thuya plicata & 1,0 \\
Tilia ssp & 3,1 \\
Ulmus ssp & 4,2 \\
\hline
\end{tabular}

Le pourcentage indique le rapport du nombre d'États ayant mentionné l'espèce comme faisant l'objet d'un programme de conservation à long terme au nombre total des Etats ayant répondu à l'enquête.

Les responsables nationaux du suivi de la résolution 2 (direction des forêts des Ėtats, Instituts de recherches forestières) considèrent que seulement quelques très rares espèces d'arbre sont menacées en tant qu'espèce. Parmi elles, les ormes (genre Ulmus) et le sapin de Sicile (Abies nebrodensis) apparaissent au premier rang. 


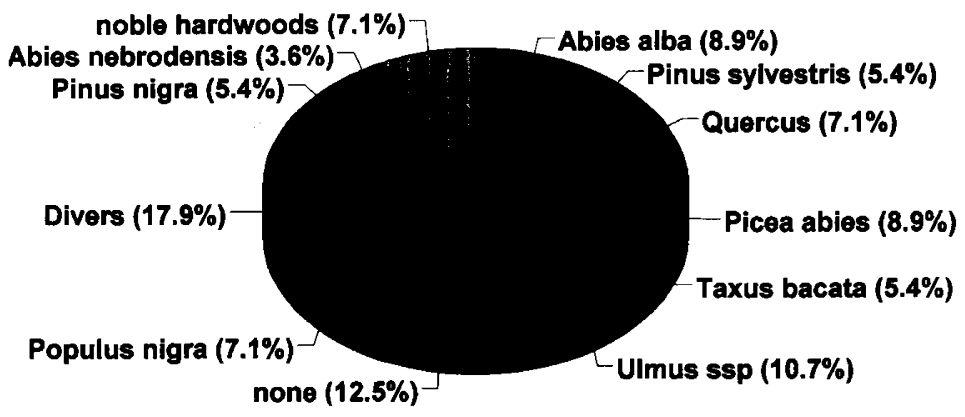

Fig 1. Enquête internationale sur l'état des ressources génétiques forestières : espèces forestières menacées dans leur diversité génétique (au niveau race ou écotype).

Le pourcentage indique le rapport du nombre d'États ayant mentionné l'espèce comme menacée, au nombre total des États ayant répondu à l'enquête.

Mais de très nombreuses espèces sont déjà sinistrées dans leur diversité génétique, et certaines de leurs populations ou de leurs écotypes originaux, sont menacés d'hybridation ou de disparition.

C'est notamment le cas de l'épicéa commun (cité comme menacé dans $8,9 \%$ des réponses); certaines populations d'Europe centrale sont très atteintes par les pluies acides et ne se régénèrent plus (Beskides polonaises, Sudètes polonaises et tchèques). Grossièrement et pour les mêmes raisons, les populations de sapin pectiné (Abies $a l b a)$ sont déclarées menacées avec le même pourcentage de réponses $(8,9 \%)$.

Les populations de chêne semblent faire les frais des politiques d'intensification forestière (conversion de taillis, remplacement par des espèces plus productives) ou d'hybridations incontrôlées avec des origines introduites. La diversité génétique inter-population apparaît menacée dans 7,1\% des États. La situation la plus préoccupante semble celle des populations de chêne liège (Quercus suber) éprouvées par les phénomènes de dépérissement généralisé qui sévissent en Afrique du Nord et dans le Sud-Ouest de l'Europe.

Le peuplier noir (Populus nigra) est cité dans $7,1 \%$ des réponses comme menacé dans sa diversité génétique par l'aménagement des cours d'eau et les hybridations avec les hybrides euraméricains.

Au même niveau d'urgence sont cités les feuillus précieux disséminés $(7,1 \%)$ souvent victimes de l'évolution progressive de la sylviculture et du remplacement des forêts mélangées et des anciens traitements (taillis sous futaies) par des futaies monospécifiques et des plantations résineuses.

D'autres espèces, plus rares mais très menacées, sont également citées (Taxus baccata notamment).

Dans l'ensemble, on observe donc une concordance satisfaisante entre les espèces choisies pour les premiers réseaux de conservation et les priorités exprimées par les représentations nationales.

Ce type d'enquête est cependant générateur d'effets pervers dont il faut être conscient. Il est clair par exemple qu'une espèce à très large répartition géographique modérément menacée sur la plus grande partie de son territoire, semblera réclamer 
des mesures plus urgentes qu'une espèce plus localisée, même menacée d'extinction, si cette menace n'est exprimée que par un ou 2 États.

Les menaces de disparition ou d'hybridation incontrôlée des populations ou des écotypes locaux sont souvent le fait. d'activités humaines (sans précision supplémentaire : $16,2 \%$; pollution génétique : $13,5 \%$; régénération artificielle : $10,8 \%$; pollution atmosphérique : $10,8 \%$; incendies de forêt : $5,4 \%$ ) ou d'origine phytosanitaire (maladies : $13,5 \%$; insectes et gibier : $5,4 \%$ ) $; 24,3 \%$ des réponses ne faisaient état d'aucune cause connue.

Trente-sept espèces d'arbres forestiers feraient l'objet d'un programme de conservation à moyen ou long terme, $80 \%$ de ces espèces sont des espèces autochtones. Les espèces le plus souvent citées sont : Picea abies, Abies sp, Pinus sylvestris, Quercus robur, Fagus sylvatica, Pinus nigra, probablement en raison de leur très large répartition géographique et de leur importance écologique et économique. Le genre Ulmus, très sévèrement affecté par les dépérissements causés par Ceratocystis ulmi, est très souvent cité. Des recoupements avec la littérature et les mesures réglementaires des États, amènent néanmoins à se demander si bon nombre de ces prétendus programmes spécifiques de conservation ne se confondent pas souvent avec les programmes nationaux de conservation de la nature (réserves et parcs nationaux), les peuplements porte-graines sélectionnés ou les collections ex situ établies dans le cadre des programmes d'amélioration en cours. La plupart des réserves génétiques in situ assument aussi d'autres fonctions (production des graines, production de bois, réserve de nature).

Les programmes nationaux spécifiquement orientés vers la conservation à moyen ou long terme d'une espèce donnée sont encore en nombre limité et souvent récents. Dans ce domaine, les pays scandinaves, l'Allemagne et la France (Arbez, 1992; Gussone et Kleinschmit, 1989; Collectif NEJS 1992), seraient les plus avancés. La plupart des États concernés par la conservation des ressources génétiques de l'épicéa commun se déclarent prêts à participer à un réseau coopératif de conservation.

Cette enquête a fait ressortir l'existence de nombreux textes réglementaires nationaux, non analysés faute de traduction et de compétence juridique. Peu de pays semblent cependant déjà disposer de réglementations spécifiques dans le domaine de la conservation.

\section{LES PREMIERS RÉSEAUX PILOTES DE CONSERVATION}

Les premiers réseaux de conservation devaient répondre à des objectifs de nature différente, contradictoires parfois :

- concerner des espèces menacées dans leur diversité génétique;

- représenter des situations très différentes, notamment vis-à-vis du modèle de répartition spatiale et du système de reproduction;

- intéresser différentes régions d'Europe;

- apporter l'illustration d'une démarche claire et convaincante, susceptible d'amorcer une dynamique de conservation à l'échelle européenne.

Le choix des 4 premières espèces ou groupe d'espèces est un compromis entre les principes précédents. 


\section{L'épicea commun (Picea abies)}

Il possède une très vaste aire de répartition dans le nord et le centre de l'Europe. Il constitue des peuplements denses, en plaine dans le nord et en montagne dans le sud (jusqu'à $2000 \mathrm{~m}$ dans les Alpes). La pollinisation est anémophile. Par les surfaces couvertes, l'importance économique et écologique de l'espèce est considérable. On observe des dépérissements importants, attribués à la pollution atmosphérique en Europe centrale et des mesures urgentes de conservation s'imposent. Il existe des dispositifs anciens d'étude de la variabilité génétique intra- et inter-population, permettant de proposer d'emblée des dispositifs d'échantillonnage et de conservation de cette variabilité fondés sur des bases scientifiques solides.

\section{Le chêne liège (Quercus suber)}

C'est une espèce feuillue sempervirente à répartition naturelle méridionale. Son importance pour l'économie et l'aménagement rural peut être capitale dans certaines de ces régions (Sardaigne, Andalousie, Alentejo, Mamora). L'espèce est aujourd'hui frappée par un dépérissement d'origine complexe, aussi bien en Europe qu'au Maghreb. Le chêne liège, à l'instar des autres Fagacées, est une espèce sociale à pollinisation anémophile. Bien qu'on sache encore peu de choses sur l'amplitude et la structuration de sa variabilité génétique, il faut d'urgence analyser et conserver l'essentiel de cette diversité. Ce programme de conservation devrait être étendu aux chênes caducifoliés d'Europe moyenne (Quercus petraea, Quercus robur) aujourd'hui beaucoup mieux connus.

\section{Le peuplier noir (Populus nigra)}

Il constitue un modèle original : il possède une répartition linéaire le long des cours d'eau, il est diö̈que (les sexes sont séparés sur des individus distincts) et la pollinisation est anémophile. Les populations locales sont l'objet d'hybridations naturelles incontrôlées par les cultivars euraméricains introduits à proximité. L'aménagement des cours d'eau (barrages, berges) est une autre cause importante de disparition des populations originelles. Économiquement, l'espèce est une source de gènes d'adaptation importante (résistance aux maladies notamment) pour la création de nouveaux hybrides. Les connaissances sur la variabilité génétique sont réelles mais encore fragmentaires, les efforts de conservation ex situ, parfois anciens, sont incomplets et encore insuffisamment coordonnés.

\section{Les feuillus précieux disséminés}

On distingue sous ce vocable général de nombreuses espèces à bois précieux, poussant sous forme d'individus isolés ou de bouquets. Les Rosacées (Prunus, Sorbus, Malus, Pirus) constituent un sous-ensemble plus homogène caractérisé notamment par une pollinisation entomophile. Ces espèces à fruits sont particulièrement importantes pour le maintien de la biodiversité animale. Leur diversité génétique est gravement menacée par l'évolution générale de la sylviculture et le remplacement progressif des forêts mélangées par des forêts monospécifiques traitées en futaie 
régulière. Des mesures de sauvegarde s'imposent. Leurs retombées concernent à la fois le forestier et le sélectionneur de variétés fruitières.

\section{DES RECHERCHES NÉCESSAIRES}

Le principal objectif concerne le maintien ou l'amélioration du potentiel d'adaptation des espèces, face aux évolutions imprévisibles de l'environnement et des besoins des hommes. La survie à long terme des forêts, comme la continuité des progrès génétiques, en dépendent (Arbez, 1984; Namkoong, 1988; Ledig 1991). Compte tenu de l'urgence, des mesures immédiates de conservation sont mises en œuvre, en dépit de connaissances scientifiques souvent réduites ou fragmentaires. Simultanément, il appartient donc à la recherche d'éclairer ces zones d'ombre. Elle sont encore nombreuses.

La connaissance des mécanismes naturels d'entretien de la diversité génétique, de l'échelle moléculaire à celle de l'écosystème forestier, devrait permettre d'imaginer des méthodes de gestion plus efficaces de cette diversité.

Pour une espèce et une population données, des informations concernant l'évolution de la diversité génétique en fonction des différents facteurs, apporteraient de précieuses indications pour gérer les forêts de production tout autant que les réserves génétiques. Ces facteurs concernent la taille de la population, son âge, le niveau de compétition entre individus, la surface des unités de régénération naturelle et le nombre des semenciers. L'effet des méthodes d'aménagement et des pratiques sylvicoles (éclaircies, régénération) sur la diversité génétique des populations forestières reste encore à mesurer.

Il faut être rapidement capable de prévenir les dépérissements forestiers de grande ampleur que pourrait provoquer un réchauffement climatique trop rapide (Gravenhorst, 1991; Peters, 1990). Des déplacements artificiels de populations vers le sud, pour palier la lenteur des migrations et accélérer l'adaptation, apportentils une réponse satisfaisante à la question posée? La plantation de populations plus méridionales dans les forêts du nord n'est-elle pas une parade plus efficace et plus rapide? L'étude approfondie du fonctionnement écophysiologique et des performances des races géographiques d'une même espèce installées anciennement en plantations comparatives dans des conditions climatiques très contrastées, apportera des informations irremplaçables pour résoudre ce problème. De tels dispositifs anciens existent pour certaines espèces, l'épicea commun (Picea abies) notamment.

Les résultats de ces expérimentations vont très au-delà des simulations possibles, ils prennent en compte les interactions importantes avec les maladies et les insectes ravageurs à la vitesse d'adaptation incomparablement plus rapide.

Lorsque l'importance économique ou écologique de l'espèce le justifie, la conservation ex situ combinée à un schéma de sélection récurrente multilignées peut être une alternative intéressante à considérer (Namkoong, 1988).

Sans imaginer des recherches nouvelles, le simple transfert aux espèces forestières des méthodes qui ont fait leur preuve chez les plantes cultivées, comme celles permettant de constituer des collections réduites et représentatives de la diversité génétique (core collections), constituerait un progrès certain (Brown, 1989). 


\section{VERS UN PROGRAMME DE CONSERVATION DES RESSOURCES GÉNÉTIQUES FORESTIÈRES EUROPÉENNES (EUFORGEN)}

La seconde conférence ministérielle, tenue à Helsinki en juin 1993 sur la protection des forêts en Europe vient d'approuver le principe d'un programme de conservation des ressources génétiques forestières européennes, proposé par l'IBPGR et le département forestier de la FAO.

Cette structure s'inspire du réseau coopératif européen de conservation des ressources génétiques des plantes cultivées (ECP/GR) qui fonctionne déjà sous l'égide de l'IBPGR.

Le projet forestier comprend :

- un secrétariat international hébergé par l'IBPGR à Rome;

- un comité exécutif composé de 2 représentants de chacun des 2 organismes fondateurs, IBPGR et FAO; ce comité exécutif est placé sous la responsabilité d'un coordinateur appartenant à l'IBPGR, chargé d'assurer la liaison entre les différents réseaux de conservation par espèces et entre les coordinateurs nationaux, d'organiser les réunions de coordination et de préparer les rapports correspondants;

- un comité de pilotage, constitué des coordinateurs nationaux désignés par les pays membres, se réunit tous les 3 ans pour évaluer la réalisation technique et financière du programme en cours, faire des recommandations pour le futur programme, et approuver le budget correspondant.

Dans une première phase, le programme européen de conservation des ressources génétiques forestières reprend le projet de réseau par espèce initié par le comité de suivi de la résolution 2 de la conférence de Strasbourg. Il comprend donc 4 réseaux intéressant respectivement l'épicea commun (Picea abies), le chêne liège (Quercus suber), le peuplier noir (Populus nigra) et les feuillus précieux.

Les participants d'un réseau donné se réunissent chaque année, et décident des actions d'évaluation et de conservation à engager ensemble, ils élaborent ce programme de collaboration, ils identifient les priorités et les besoins de recherche, ils s'accordent sur l'opportunité, l'organisation et le fonctionnement des bases de données et préparent des listes de descripteurs par espèce.

Un organisme est choisi par les participants de chaque réseau, en fonction de sa compétence technique et de la volonté d'engagement du pays concerné, pour créer et administrer la base de données des ressources génétiques de l'espèce ou du groupe d'espèces.

Les États intéressés par l'adhésion à un ou plusieurs réseaux de conservation signent un contrat avec l'IBPGR fixant le montant de leur participation financière au budget de coopération et l'ampleur des moyens en personnel éventuellement mis à disposition du programme commun (création et maintenance de la base de données, gestion des banques de semence...). Le budget global de fonctionnement du programme et la cotisation annuelle des États sont actuellement en cours de discussion.

L'aval sans réserve donné à ce projet par les ministres de l'Agriculture et des Forêts réunis à Helsinki, permet de poursuivre, en principe sans interruption, le programme de conservation initié par le comité de suivi de la résolution 2 de la conférence de Strasbourg. 


\section{CONCLUSION}

Dans les décennies d'après guerre, la fonction de production de la forêt est par priorité considérée et l'opinion publique n'y trouve rien à redire : les plantations monospécifiques d'essences à croissance rapide constituent alors le modèle dominant. Des erreurs et des excès dans ce sens, comme le goût croissant des citadins pour la nature sauvage inversent aujourd'hui cette tendance, l'opinion publique se préoccupe de paysages et le scientifique de gestion durable. Les concepts de ressources génétiques et de biodiversité font leur apparition en forêt. Si de très nombreuses espèces disparaissent en forêt tropicale humide à la faveur de la surexploitation et des défrichements, dans la zone tempérée le danger immédiat est d'une autre nature, ce sont surtout les écotypes et les races locales qui se trouvent menacés par l'intensification de la sylviculture et les transferts artificiels de semence et de plants sur de grandes distances. Des mesures d'urgence s'imposaient donc pour conserver la variabilité génétique intra- et inter-spécifique actuelle.

Ces mesures d'urgence commencent à se mettre en place de manière coordonnée, sous l'égide de la conférence de Strasbourg, aujourd'hui relayée par l'IBPGR et la FAO. Elles concernent dans un premier temps quelques espèces forestières de grande importance écologique et économique dont la diversité génétique inter-population apparaît menacée : l'épicea commun, le peuplier noir, le chêne liège et les feuillus précieux. Bien que validé par une enquête internationale, ce choix d'espèces peut toujours être discuté. Là n'est pas la question, il s'agit beaucoup plus d'initier et de pérenniser une dynamique internationale de conservation.

L'objectif choisi, maintenir ou augmenter l'adaptabilité des espèces et des populations vis-à-vis des changements imprévisibles de l'environnement, réunit la plupart des suffrages. De nombreuses recherches restent cependant nécessaires pour y parvenir efficacement. Elles concernent l'analyse de la variabilité génétique de nombreuses espèces et l'étude de leur système de reproduction, les mécanismes d'entretien de la variabilité génétique en forêt naturelle, les méthodes d'échantillonnage et de gestion dynamique de cette variabilité génétique utilisables par le sélectionneur.

Les mesures prises en application de la conférence de Strasbourg font une large place à la conservation in situ, c'est faire l'hypothèse implicite que les changements climatiques attendus seront compatibles avec la survie sur place de la plupart des populations actuelles. Qu'en sera-t-il exactement?

La simulation des scénarios climatiques attendus dans les différentes régions et la prédiction correspondante du comportement local des grandes espèces est intéressante mais problématique. On connaît mal la capacité et la vitesse d'évolution d'une espèce unique face à un changement climatique donné, on s'avère par ailleurs incapable de prévoir ce qui se passera lorsque 2 espèces d'arbre cohabiteront dans la même forêt, ou d'imaginer l'interaction de l'une d'entre elles avec les insectes et les maladies de son environnement si la température moyenne annuelle vient à augmenter de quelques degrés. C'est dire que la méthodologie de conservation in situ des ressources génétiques a beaucoup à gagner d'une meilleure connaissance du fonctionnement des écosystèmes forestiers.

Quels que soient les progrès attendus dans ce domaine, rien ne remplacera pourtant l'analyse et le suivi temporel des plus vieilles expériences multi-stationnelles de 
provenances et de descendances (épicea commun, hêtre...). Ces analyses renseigneront notamment sur l'allure et la rapidité des modifications de structure génétique auxquelles il faut s'attendre, en transférant les populations de plusieurs centaines ou de plusieurs milliers de kilomètres vers le nord ou vers le sud.

Il n'est cependant pas interdit de penser que dans certaines situations (hautes latitudes, hautes altitudes), le réchauffement climatique attendu ne permettra pas la survie sur place des populations. Il en va de même dès aujourd'hui dans certaines régions, en raison de la pollution atmosphérique. Force alors est de recourir à une sauvegarde ex situ, prolongée lorsque l'importance écologique ou économique de l'espèce le justifie, par une méthode de conservation dynamique, bien adaptée à la biologie de l'espèce. Des recherches dans ce sens doivent encore être amorcées chez les arbres forestiers.

Pour des raisons de faisabilité immédiate, les mesures de conservation actuelles sont engagées espèce par espèce, en commençant par des espèces économiquement ou écologiquement importantes, aptes à sensibiliser les décideurs. Restent la majorité des autres espèces forestières, secondaires, mineures ou rares et plus exposées par conséquent.

Des réseaux de conservation des écosystèmes forestiers représentatifs seraient donc le complément indispensable de la conservation espèce par espèce pour les espèces forestières secondaires ou rares. À la condition, cette fois encore, d'une survie en place possible dans le cadre des scénarios de changements climatiques attendus.

\section{REMERCIEMENTS}

L'enquête mentionnée dans cet article sur l'état des ressources génétiques forestières en Europe et leurs programmes nationaux de conservation, a bénéficié de l'aide financière de la commission des Communautés européennes et du département des forêts de la FAO.

\section{RÉFÉRENCES}

Arbez M (1984) L'avenir des forêts dépend d'une gestion raisonnée de leurs ressources génétiques. In: Symposium IUFRO «Impacts de l'homme sur la forêt», Strasbourg (France), INRA Service des publications, Paris

Arbez M (1992) Un programme national de conservation des ressources génétiques forestières. In: Conservation et gestion des ressources génétiques végétales en France, BRG et CTPS, Paris, 33-43

Brown AHD (1989) Core collection: a practical approach to genetic resources management. Genome 31, 818-824

Forest Genetic Resources Working Group (1992) A report on gene resources of forest trees NEJS (Nordisk Ministerrad)

Ministry of Agriculture and Forestry Conference Secretariat (1993) Report on the follow-up of the Strasbourg resolutions. Helsinki, Finland

Gravenhorst G (1991) Genetic variation in forest tree populations: the view point of bioclimatologist on genetic variation of forest trees. In: Genetic variation in European population of forest trees (Müller Starck F, Ziehe M, eds). Sauerlander's Verlag, Frankfurt am Main, 2-19 
Gussone HA, Kleinschmit J (1989) Konzept zur Erhaltung forstlicher Genressourcen in der Bundesrepublik Deutschland. Forst und Holz 15, 379-404

Hamrick JL, Godt MJW (1990) Allozyme diversity in plant species. In: Plant population genetics, breeding and genetic resources (Brown AHD, Clegg MT, Kahler AL, Weir BS, eds). Sinauer Associates, Sunderland, MA, 43-63

Ledig FT (1991) The role of genetic diversity in maintaining the global ecosystem. In: Proceedings from the 10th World Forestry Congress, Paris-RFF Nancy, vol 2, 71-78

Namkoong G (1988) Population genetics and the dynamics of conservation. In: $L$ Knutson and AK Stoner Beltville Symposia in Agricultural Research (13) Biotic Diversity and Germplasm Preservation, Global imperatives, mai 1988. Kluwer Academic Publisher, Dordrecht, the Netherlands, 161-181

Palmberg C, Esquinas-Alcazar JT (1990) The role of the United Nations agencies and the other international organizations in the conservation of plant genetic resources. For Ecol Manage 35, 1-2, 171-197

Peters RL (1990) Effects of global warming on forests. For Ecol Manage 35, 1-2, 13-33

Ziehe $\mathrm{M}$ et al (1987) Gene resources and gene conservation in forest trees: General concept. In: Genetic effects of pollutants in forest tree populations. Proceeding IUFRO Springer-Verlag, 173-185 